Archives

5 | 1990

Varia

\title{
Comment prouver la mort du roi. Le cas de Sébastien de Portugal
}

\section{Lucette Valensi}

\section{OpenEdition}

Journals

Édition électronique

URL : http://journals.openedition.org/ccrh/2879

DOI : $10.4000 /$ ccrh.2879

ISSN : $1760-7906$

Éditeur

Centre de recherches historiques - EHESS

Édition imprimée

Date de publication : 15 avril 1990

ISSN : 0990-9141

Référence électronique

Lucette Valensi, «Comment prouver la mort du roi. Le cas de Sébastien de Portugal », Les Cahiers du Centre de Recherches Historiques [En ligne], 5 | 1990, mis en ligne le 20 mars 2009, consulté le 19 avril 2019. URL : http://journals.openedition.org/ccrh/2879; DOI : 10.4000/ccrh.2879

Ce document a été généré automatiquement le 19 avril 2019

Article L.111-1 du Code de la propriété intellectuelle. 


\title{
Comment prouver la mort du roi. Le cas de Sébastien de Portugal
}

\author{
Lucette Valensi
}

1 Peut-être mon intervention arrive-t-elle trop tard, puisque un quotidien marocain $\mathrm{du}$ mois d'août dit nous apporter enfin la preuve définitive que Sébastien de Portugal est bien mort le 4 août 1578 au cours de la fameuse bataille des Trois Rois qui opposa l'armée portugaise et son allié, le sultan déchu Muhammad al-Mutawwakil, à l'armée marocaine commandée par le sultan Abdelmalek. Les Marocains sortaient victorieux de la bataille, mais le sultan Abdelmalek était mort de maladie au cours de l'affrontement, de même qu'al-Mutawwakil (qui périt noyé, alors qu'il essayait de s'enfuir). Quant à Sébastien, il disparut dans la mêlée.

2 Le problème que j'essayais de résoudre avant de recevoir le quotidien marocain que je viens de citer est celui de savoir comment les différents protagonistes prouvèrent, les uns, que le roi Sébastien était mort dans la bataille, les autres, qu'il avait survécu. Ces protagonistes forment trois ensembles que je désignerai, pour simplifier, comme marocain pour le premier, portugais pour le second, et espagnol pour le troisième. L'Espagne, en effet, était directement intéressée par l'issue du conflit, car le chaste Sébastien étant mort sans descendance, le Portugal tombait sous la domination de Philippe II deux ans après la défaite de 1578, et il ne devait retrouver son indépendance qu'en 1640. Je décrirai ici les trois systèmes de preuves, et commencerai par le Maroc.

\section{Les marocains veulent apporter la preuve que Sébastien est mort}

3 De solides motifs politiques conduisent le nouveau sultan, Ahmad al-Mansûr, à démontrer fermement la mort de Sébastien. Son frère, le sultan décédé, n'avait pas voulu cet affrontement avec l'armée portugaise, et il avait usé de tous les moyens diplomatiques et politiques pour l'éviter. Vainqueur, Ahmad al-Mansûr avait tout intérêt à suspendre les hostilités et à trouver un terrain d'entente avec les Ibériques. Le plus urgent pour lui était - après avoir été acclamé sultan sur le champ de bataille, - de se faire reconnaître par 
l'ensemble du pays en se présentant lui-même, comme c'était l'usage au Maroc, devant les tribus et aux portes des villes.

Il recourt d'abord à la preuve tangible pour attester la mort de Sébastien. Le 5 août, au crépuscule, il fait reconnaître le cadavre du roi sur le champ de bataille par des Portugais qui avaient été ses serviteurs et fait ensuite transporter le corps sous sa tente. Suit une épreuve d'authentification: des nobles portugais faits prisonniers lors de la bataille doivent identifier la dépouille posée nue aux pieds du sultan. Il semble que cette épreuve d'authentification ait été répétée trois jours plus tard (Luis de Oxeda). Après quoi, une série de mesures réalisant les directives du sultan renouvellent et renforcent les preuves de la mort du roi : 1. Le cadavre de Sébastien est mis en bière dans de la chaux vive, ce qui lui assurerait une plus longue conservation et autoriserait de nouvelles identifications. 2. Le cercueil est transporté scellé à El Ksar, sous escorte marocaine - ce qui est normal -, mais aussi accompagné de nobles portugais, qui pourraient donc témoigner que c'était bien le roi qu'on avait déplacé. 3 . Là, des captifs portugais procèdent à l'inhumation, dans le château, après une veillée et une messe funéraires. Ces rituels, toujours assurés par des chrétiens portugais, confirment donc la mort du roi. 4. L'un des deux grands officiers marocains qui avaient escorté la dépouille du roi jusqu'à El Ksar se rend à Tanger, toujours tenue par les Portugais, pour leur annoncer officiellement le résultat de la bataille et l'inhumation du roi à laquelle il avait personnellement veillé. Cette annonce solennelle clôt, du côté marocain, la première séquence des opérations d'authentification de la mort du roi portugais.

En décembre 1578, à la suite d'une action diplomatique qui ne nous intéresse pas ici, le corps de Sébastien est déterré pour être transporté d'El Ksar à Ceuta. De nouveau, le transport se faisant en compagnie de nobles portugais rachetés de leur captivité, ils pourraient, le moment venu, témoigner de la bonne foi du sultan marocain et de l'identité de la dépouille qu'ils accompagnaient. A Ceuta, place tenue par les Portugais, les agents du sultan remettent le corps au gouverneur, qui en confie la garde aux frères trinitaires. C'est la déclaration solennelle prononcée alors par le gouverneur que Le Matin du Sahara reproduit quatre siècles plus tard.

6 Pour les Marocains, le but qu'ils poursuivaient est atteint dès le 6 décembre 1578. Ils n'auront pas, désormais, à revenir sur le sort de Sébastien. Les preuves ultérieures que nous trouvons $\mathrm{du}$ côté marocain (ou plus largement, musulman) sont d'ordre documentaire. Il s'agit de récits de la bataille faits dans le cadre de chroniques de l'époque et du règne d'al-Mansûr. Mais on trouve aussi, sur le site de la bataille, la base d'une stèle érigée par les Portugais dans les années 1930, et tombée en ruine depuis. Les habitants du lieu la désignent comme la tombe de Sébastien, proposant donc encore une preuve - monumentale et apocryphe - de la mort du roi portugais.

\section{Les espagnols (et certains portugais) veulent et doivent prouver la mort de Sébastien}

7 La nouvelle du désastre étant parvenue dans la capitale portugaise quelques jours après la bataille, les gouverneurs qui exerçaient le pouvoir en l'absence du roi doivent immédiatement assurer sa succession et la continuité de l'autorité légitime. Elle revient au cardinal Henri, oncle du roi Sébastien, qu'on tire de sa retraite pour l'introniser à Lisbonne. Mais avant de proclamer roi le vieux cardinal, il faut établir officiellement que 
Sébastien est mort. L'annonce publique de la disparition du roi est faite le 24 août. Puis la cérémonie des funérailles signifie à son tour au peuple portugais la mort du souverain : le port de vêtements noirs par tous les membres du cortège et de drap noir par les équipages, le déploiement de bannières de même couleur, la rupture des écussons royaux, l'injonction répétée faite à la foule de pleurer la mort du roi, la répétition de tous ces gestes aux points stratégiques de la capitale, doivent convaincre le peuple que le roi Sébastien n'est plus.

8 Ce rituel grandiose avait lieu toutefois en l'absence du cadavre du roi. Or deux ans plus tard, le vieux cardinal Henri étant mort, ce que les Portugais redoutaient plus que tout arriva: la succession revint au roi d'Espagne Philippe II et leur pays perdit son autonomie. Tragédie nationale dont tous les documents de l'époque portent témoignage, elle fut mal supportée par les Portugais qui n'admettaient pas la légitimité du monarque espagnol. Celui-ci eut donc à la démontrer sans relâche. L'un des moyens employés fut de confirmer la mort de Sébastien: 1. Par la preuve tangible. Philippe II fait rapatrier le corps de Sébastien, qui est transporté de Ceuta à l'Algarve. 2. Par une répétition des rituels funéraires. A l'arrivée du corps, des cérémonies funèbres sont célébrées à Faro en présence de membres du haut clergé et de la noblesse. Puis le corps est transporté en grande pompe vers la nécropole royale de Belem (près de Lisbonne), suivant un itinéraire qui n'est pas le plus court. Un cortège formé des grands du royaume accompagne le cercueil recouvert d'un drap d'or et précédé d'une immense croix dorée. En chemin, un nouveau cérémonial spectaculaire a lieu. Le cortège fait halte à Evora où se trouvent les tombes des infants, fils des rois Manuel et Joao III. Ils sont déterrés pour être transportés avec Sébastien : rituel ostentatoire d'agrégation de Sébastien au monde des morts. Il est remis à sa place dans sa parenté défunte. Une messe de requiem est célébrée le 10 décembre, et de nouveau le 11, dans l'affluence du peuple et du clergé. Enfin le cortège quitte la ville d'Evora et se dirige vers Belem, où le corps de Sébastien est enseveli, en présence de Philippe II, dans le transept de l'église des Jeronimos. Sébastien a donc enfin rejoint le domicile des morts de son lignage. C'est bien la preuve qu'il est mort.

Pourtant, un siècle plus tard, après la restauration de l'indépendance du Portugal, le roi Pedro II fait encore exhumer les restes de Sébastien pour les placer dans un tombeau monumental, exacte copie de ceux des rois Manuel et Joao III, du cardinal Henri et de la reine Catherine. Une épitaphe en latin atteste qu'en ce tombeau repose Sébastien, mort sur les rivages africains.

10 Ainsi, pendant plus d'un siècle, la mise en scène réitérée des funérailles royales devaitelle affirmer et confirmer la mort de Sébastien.

Preuves concordantes : elles vinrent sous la forme des nombreuses relations de la bataille qui circulèrent, manuscrites ou imprimées, dans la Péninsule et dans le reste de l'Europe. Je ne ferai pas ici l'inventaire de ces témoignages, il suffit de savoir qu'ils sont nombreux. Nous échappent, évidemment, les récits oraux qui ont dû circuler avec les rescapés de la bataille et les captifs rachetés.

Voilà en tout cas un ensemble de preuves apportées, tant au Maroc qu'au Portugal et en Europe, de la mort du roi Sébastien. Et pourtant, elles n'ont pas convaincu. Il s'est trouvé des Portugais en grand nombre qui n'ont pas cru à la mort de leur roi. 


\section{Des portugais apportent la preuve que le roi Sébastien est vivant}

13 Ce qui me paraît le plus intéressant, c'est maintenant l'ensemble des preuves qu'apportent ces Portugais qui croient leur roi vivant. Plus intéressant, car nous avons la certitude aujourd'hui que Sébastien est mort. Nous savons donc que ces preuves ne se sont pas vérifiées. Or elles leur ont paru suffisantes, au XVII siècle surtout. A quel registre appartiennent-elles?

14 Les premières sont inséparables de celles qui démontraient la mort du roi. Elles sont de même nature et répondent au même principe: c'est l'information qui circule immédiatement après la bataille. L'information orale est relayée par la correspondance écrite - et c'est ainsi qu'elle nous parvient. Elle est probante, car aussi cohérente et vraisemblable que son contraire, et livrée dans les mêmes conditions. Par exemple, le 13 août, l'Espagnol Luis de Herrera écrit à Philippe II qu'il a appris de chevaliers faits prisonniers et dignes de foi que Sébastien mort, son corps a été placé dans un cercueil et envoyé à Fès sur ordre du nouveau souverain marocain. Mais le même correspondant écrit le même jour, au même destinataire, une lettre selon laquelle des chevaliers prisonniers ont écrit par la voie de Tétouan. Ils disent que Sébastien s'est échappé avec cinq autres cavaliers en direction d'Arzila. Il ajoute cependant que selon des Maures venus d'Arzila, on n'a aucune nouvelle du roi, « ni mort ni vif »1. A priori, rien ne permet de conclure à la véracité de l'une ou l'autre affirmation. Parfois du reste, l'information est univoque : elle annonce que Sébastien s'est échappé, ou qu'il a été fait prisonnier, sans du tout évoquer l'hypothèse de son décès. Un exemple : le 8 août, un Portugais posté au Sud du pays reçoit un courrier d'un capitaine de Ceuta, au Maroc, lequel a reçu une lettre du caïd de Tétouan, lui annonçant la défaite et la capture de Sébastien².

15 Je rangerai dans la même catégorie les récits plus tardifs qui, comme les informations immédiates, sont probants en raison du caractère vraisemblable de leur contenu, et cohérent de leurs énoncés. Le tout premier récit imprimé de la bataille qui soit dû à un auteur portugais est de 1602. Il décrit Sébastien se battant comme un lion, fonçant sur l'ennemi, puis disparaissant sans que personne ne l'ai jamais vu mort ou captif3.

Après l'information, vient l'infirmation. Ici, il s'agit de démontrer que les preuves contenues dans les thèses adverses ne résistent pas à des arguments d'ordre logique. 1. Le corps nu que le roi du Maroc fait reconnaître par des chevaliers portugais ne porte aucun insigne royal. 2. Cette reconnaissance a lieu à la nuit tombée. 3. Elle a lieu plus de vingtquatre heures après la fin de la bataille, le corps ayant été exposé longtemps à la chaleur torride du mois d'août au Maroc, il devait donc être méconnaissable. 4. Le corps présentait de nombreuses blessures à la tête; il ne pouvait donc pas être identifié. 5 . Si des prisonniers portugais affirmèrent reconnaître leur roi, c'était pour couvrir sa fuite et lui laisser le temps de se mettre en lieu sûr. 6. Ou bien c'est en raison du fait que le Sultan leur avait promis la liberté s'ils reconnaissaient leur roi $^{4}$. 7. Bien plus tard enfin, la dépouille de Sébastien ayant reçu une sépulture monumentale à Belem, une épitaphe fut gravée sur le tombeau. Or tandis qu'elle voulait signifier la mort du roi, on l'interpréta comme la preuve qu'un autre corps gisait là.

Conditur hoc tumulo, si vera est fama, Sebastus

Quem tulit in Lybicis mors properata plagis... 
"Si vera est fama », si ce qu'on dit est vrai : la formule autorisait le doute sur l'identité du corps qu'abritait le tombeau. Montalvo Machado... Au xviII ${ }^{\mathrm{e}}$ siècle encore, ceux qui espèrent le retour du roi continuent de réfuter avec les mêmes arguments la thèse de sa mort. Ainsi, par exemple, de l'auteur de la Narraçao das fundamentos que ha para affirmar a vida, e vinda del Rey D. Sebastiao outra vez a Portugal ${ }^{5}$.

Les témoignages, ou au contraire l'absence de témoignages dignes de foi, viennent corroborer ces informations. Or, aucun témoin portugais de la bataille n'a jamais dit avoir vu tomber le roi. Même des années après l'événement, les captifs qui rentrèrent du Maroc n'apportèrent aucune pièce supplémentaire au dossier toujours ouvert. A l'inverse, des Portugais déclarèrent avoir vu le roi, ou avoir vu d'autres Portugais qui avaient vu le roi après la bataille.

Preuve tangible : elle ne pouvait être fournie que par Sébastien lui-même. Et elle vint en effet, sous les traits de quatre imposteurs successifs, qui se firent passer pour Dom Sébastien, et en qui on reconnut le roi. Le premier, surgi en 1584, recruta des fidèles en milieu paysan, mais finit aux galères. Le second, surgi la même année aux environs de Lisbonne, organisa une armée et un embryon de gouvernement, mais ne résista pas à un affrontement avec les troupes régulières et finit sur le gibet. Dix ans plus tard, c'est un modeste pâtissier exerçant en Espagne qui se voit promu roi par la ferveur d'un membre du clergé portugais. Tous deux seront pendus en 1595. Quant au dernier faux-Sébastien, il s'agit de Marco Tullio Catizone, dit le Calabrais, dont l'imposture commence à Venise en 1598 et s'achève sur le gibet en septembre 1603. Mais ses partisans, qui se recrutèrent dans le clergé comme dans la noblesse portugaise en exil, continuèrent de penser que le Calabrais était bien le roi Sébastien ${ }^{6}$. Preuve de la véracité de la preuve : à ce point je devrais rapporter les arguments défendus par les partisans de ces quatre imposteurs pour démontrer qu'ils étaient Sébastien. Sur le quatrième notamment, seize particularités physiques connues par les proches familiers du roi disparu furent « reconnues » sur le corps du Calabrais. Mais on ne développera pas cette séquence.

Preuve documentaire: elle est fournie par les sentences délivrées par trois papes successifs. La première, datée de 1598, est de Clément VIII. Il y déclare que son fils aimé Don Sébastien, s'est présenté personnellement à la curie et qu'il est le roi véritable et légitime du Portugal. S'étant perdu en Afrique au camp d'Alcasar Kivir, il est resté occulte jusqu'à son retour à Rome. Le conclave et la curie ayant authentifié ses déclarations le reconnaissent comme l'héritier légitime du Portugal et somment donc Philippe III $\mathrm{d}$ 'Espagne de lui rendre ses états sans délai. Le pape Paul $\mathrm{V}$ réitère cette sentence le 17 mars 1617, suivi par Urbain VIII, qui s'adresse à Philippe IV dans les mêmes termes en janvier $1620^{7}$. Ces documents ont été reproduits inlassablement jusqu'au $\mathrm{xx}^{\mathrm{e}}$ siècle, entretenant le doute sur la date de la mort du roi Sébastien chez quelques historiens portugais. Il s'agit de faux.

Récits cohérents et vraisemblables, témoignages directs et indirects, preuves tangibles, documents, tous ces moyens auxquels des Portugais ont recours pour nier la mort du roi nous paraissent recevables, car on pouvait soumettre l'ensemble de ces preuves à des tests de contrôle et de vérification. Mais les sébastianistes qui attendaient le retour de leur roi ne se contentèrent pas de les répéter et de les combiner. Ils leur adjoignirent des preuves qui, de notre point de vue, appartiennent à un tout autre registre. Ce sont « les avis du ciel et de la terre », comme les appelle Luis Torres de Lima (1634), et ils se rangent en trois catégories : 
22 1. Ce sont d'abord les signes, ces « ambassadeurs" du futur. Les signes annoncent ce qui doit arriver; ils peuvent donc être soumis à vérification. Dans le cas de Sébastien, les signes qui avaient précédé sa naissance s'étaient vérifiés, de même que ceux qui avaient annoncé le désastre d'Alkasar Kivir - signes qu'il avait eu le tort de ne pas prendre en compte. Ces augures peuvent être les désastres naturels, ou le passage d'une comète. Puisque ces signes s'étaient vérifiés, ceux qui annonçaient le retour de Sébastien se vérifieraient de même.

23 2. Les visions et prodiges démontrent également le retour certain de Sébastien. C'est par exemple l'histoire de ce pauvre homme de Termo de Silves, dans l'Algarve, qui a eu plusieurs visions lui annonçant, en 1636, 37, 39, la venue prochaine d'un roi âgé de 103 ans, caché dans une île inconnue. Et ce roi, c'est Sébastien ${ }^{8}$. Relèvent de la même catégorie les miracles, qui ne souffrent pas d'autre interprétation que celle du retour de Sébastien. Comment comprendre en effet que dans une église portugaise la statue de saint Sébastien se couvre de sueur devant deux enfants innocents, sinon comme l'annonce de l'arrivée imminente du roi dont le saint fut le patron ${ }^{9}$ ?.

24 3. Les prophéties enfin justifient l'attente du roi. Celles de Bandarra sont les plus importantes. Proférées sous la forme de poèmes au contenu obscur, les Trovas avaient été condamnées par l'Inquisition dès 1540, mais elles n'en conservaient pas moins une grande popularité au Portugal. Elles étaient vraies, selon les sébastianistes et tout particulièrement Dom Joam de Castro (Paraphrase e concordancia de alguas prophecias de Bandarra, çapateiro de Trancoso), parce qu'elles concordaient avec d'autres prophéties anciennes et modernes d'une part ; parce qu'elles s'étaient conservées d'autre part. Or ces Trovas avaient prévu l'arrivée du roi désiré et elles annonçaient son retour. D'autres prophéties, postérieures à la disparition de Sébastien, telle celle de Bartolomeu Vaz Pincho $\mathrm{au} \mathrm{XVII}{ }^{\mathrm{e}}$ siècle, décrivent avec précision selon quel scénario le roi dissimulé fera son apparition ${ }^{10}$. La vérité des prophéties étant prouvée par d'autres prophéties, on ne se lassa pas de convoquer les prophéties des saints les plus divers, en vers et en prose, antérieures au règne de Sébastien ou postérieures à sa disparition ${ }^{11}$.

L'accumulation de preuves la plus systématique est apportée par le fameux père jésuite Antonio Vieira. On sait que cette puissante figure $\mathrm{du} \mathrm{XVII}^{\mathrm{e}}$ siècle portugais a fougueusement défendu l'idée que le Portugal avait vocation à fonder le cinquième empire annoncé par la prophétie de Daniel. L'avènement de la monarchie universelle était imminent. Mais contre les sébastianistes, Vieira crut d'abord que Joao IV, restaurateur de l'indépendance du Portugal en 1640, était le roi occulte Encoberto, dont on attendait la venue. Par la suite, après les échecs et le décès de ce roi, Vieira désignera ses successeurs pour remplir la fonction de monarque universel ${ }^{12}$. Mais on trouve à la suite de ses Espérances du Portugal, Cinquième Empire du monde, écrit au Brésil en 1659, un traité prouvant que Sébastien est vivant ${ }^{13}$.

Vieira annonce qu'il recourra aux huit preuves suivantes pour le démontrer : 1. la raison et les conjectures, 2. les prophéties, 3. les révélations, 4. les prodiges, 5. les pronostics de grands astrologues, 6. les écrits historiques, 7. les jugements politiques, 8. les traditions des mahométans. Je reprendrai rapidement ici quelques exemples de ces preuves.

Preuve logique, relevant de la première catégorie qu'il distingue : Dieu peut tout; donc des individus peuvent avoir une très longue vie. Preuve rationnelle encore : la mort de Sébastien n'a jamais été prouvée. Ou encore : on a le témoignage de gens qui l'ont vu à Venise, notamment Joao de Castro. 


\section{NOTES}

1. SIHM, Angleterre, t. I, 1918, pp. 322-325. Mêmes informations contradictoires à Paris, fin août, à Londres, fin septembre ; à Istanbul, début novembre.

2. SIHM, Espagne, t. III, pp. 453-457.

3. Joao de CASTRO, Discurso da vida...., 1602, p. 27 verso.

4. Voir NIETO, OXEDA, PALMA CAYET, Agrippa d'AUBIGNE, etc., in SIHM ; Joao de CASTRO, op. cit., p. 28 verso, etc.

5. BN Lisbonne, Mss. cod $11.379,61 \mathrm{f}$.

6. Miguel d'ANTAS, Les faux Don Sébastien. Étude sur l'histoire du Portugal, Paris, 1866 ; HERRERA, Segunda parte de la Historia general del mundo, Madrid, 1601 ; Luis de TORRES DE LIMA, Compendio das mas notaveis cousas no reyno de Portugal..., Lisbonne, 1722 (1ère éd., 1654); Joao de CASTRO, Discurso da vida do sempre bem vindo et apparecido Rey Dom Sebastiam nosso senhor..., Paris, 1602.

7. Bibliothèque Ajuda, Lisbonne, Movimento do Orbe Lusitano, t. 2, 50-V-35, fol. 62-63. BN Lisbonne, Cod. 401, Sentença e confirmaçoens...

8. BN Lisbonne, Mss. cod. 551, fol. 136 sq. Autres visions dans le même recueil.

9. BN Lisbonne, Mss. cod 401.

10. Bibl. Ajuda, Lisbonne, vol.50-V-30, fol. 216. Voir également la Narraçao..., texte du XVIII siècle, cité plus haut.

11. Voir notamment BN Lisbonne, mss. cod 551, Colleçao de escriptos sebastianistas. cod. 627, Profecias...

12. Sur Vieira, voir notamment Raymond CANTEL, Prophétisme et messianisme dans l'oeuvre d'Antonio Vieira, Poitiers, 1960.

13. BN Lisbonne, cod. 400 , fol. 245-280.

Les Cahiers du Centre de Recherches Historiques, 5 | 1990 


\section{AUTEUR}

\section{LUCETTE VALENSI}

Lucette VALENSI est directeur d'études au Centre de Recherches Historiques.Le matin du Sahara et du Maghreb, (jeudi 24 août 1989, p. 3)Sébastien mort à la bataille de Oued El Makhazine : personne désormais ne saurait le contester en prenant connaissance du document que nous publions et par lequel des officiels portugais déclarent officiellement « réceptionner » la dépouille de Sébastien que le roi Moulay Ahmed Eddahbi, frère de Moulay Abdelmalek, leur a restituée avec la consigne de la remettre au gouvernement de Sebta, alors sous occupation portugaise. 